\title{
LÓGICA Y RAZÓN
}

JOSÉ FERRATER MORA

Bryn Mawr College

Desde Aristóteles se ha planteado el problema de si la lógica -el "arte lógico"- es, como dicho autor propuso, una especie de propedéutica de la filosofía y, en general, de todo conocimiento, o si es, como afirmaron los estoicos, una "parte" de la filosofía. Quedan aún autores que mantienen una u otra de estas posiciones. Así, por ejemplo, William Kneale ha propuesto que "si la filosofía es prudente, admitirá la lógica como miembro de su familia con todos los derechos, no tanto con la esperanza de obtener algunos favores excepcionales para los demás miembros como con el propósito de subsanar una vieja injusticia y acrecentar su propia reputación entre gentes de buen juicio". ${ }^{1}$ La principal razón de esta propuesta es "la creencia de que algunas de las cuestiones más importantes de la lógica son filosóficas más bien que matemáticas". ${ }^{2}$ Por otro lado, autores como Bertrand Russell creyeron por un tiempo que los problemas filosóficos son, a la postre, problemas lógicos, de modo que la clase de lógica que se adopte depende de la clase de filosofía que se haga. ${ }^{s}$ Hay quienes siguen pensando que la lógica puede ser una propedéutica para la filosofía, pero esto puede interpretarse a su vez de dos maneras: concibiendo la lógica como una especie de "ciencia básica", dentro de cuyos

1 William Kneale, "Symposium: What Can Do Logic for Philosophy?" en Proceeding of the Aristotelian Society, Supp. Vol. 22 (1948), p. 156.

2 Loc. cit. Desde 1948 William Kneale puede haber cambiado de opinión al respecto, pero su obra (en colaboración con Marta Kneale) sobre "el desarrollo de la lógica" parece ser una larga ilustración de la opinión citada.

3 B. Russell, Our Knowledge of the External World (London, 1914, ed. rev., 1926), Cap. II ("La lógica como esencia de la filosofía”). 
esquemas formales deben tratarse todas las cuestiones filosóficas, o como un lenguaje en el cual hay que expresar los argumentos filosóficos si se espera que éstos tengan validez formal. Hay también quienes sostienen que la lógica no es filosóficamente neutral, ya que su desarrollo depende de supuestos filosóficos, y también quienes mantienen la completa neutralidad de la lógica con respecto a cualesquiera posiciones filosóficas.

La posición que va a mantenerse aquí son, en rigor, dos posiciones: (1) La lógica formal es neutral en el sentido de ser independiente con respecto a cualquier "contenido", es decir, a cualquier "sujeto" o "tema", siendo tal neutralidad mayor aun que la neutralidad e independencia res. pecto a cualquier "contenido", "sujeto" o "tema" de ciertas expresiones del lenguaje corriente tales como ' $y$ ', 'o', etc. (2) Pueden plantearse, de todos modos, problemas filosóficos en la lógica; en todo caso, algunas de las nociones usadas en lógica pueden servir de "modelo", sea como punto de partida para el análisis de cuestiones filosóficas, sea a modo de "arsenal conceptual" al que puede acudirse en el curso de dicho análisis."

Esto quiere decir que no se considera aquí la lógica ni como "parte" ni como "propedéutica" de la filosofía, pero sí como manteniendo con la filosofía "relaciones especiales".

Los filósofos -incluyendo los que han entendido el análisis filosófico primariamente como "análisis lógico"- no son, en tanto que filósofos, lógicos puros. "Sólo a los lógicos puros -que son muy pocos", escribe Jesús Mosterín-, "interesa la lógica por sí misma". ¿En qué se interesan los que no son lógicos puros? Según Mosterín, sobre todo

- El propio Kneale, en el artículo citado, se inclina por esta posición al poner de relieve que al hacer filosofía "tenemos a menudo ocasión de usar términos técnicos de la lógica. Cuando, por ejemplo, discutimos el fenomenalismo, hablamos mucho de proposiciones hipotéticas" (art. cit., pág. 160). Esta opinión puede mantenerse sin necesidad de penear que la lógica es "un miembro de la filosofía con plenos derechos".

5 Jesús Mosterín, Lógica de primer orden (Barcelona, 1970), p. 7. 
en las aplicaciones de la lógica, las cuales consisten en sa. ber probar que una sentencia dada es o no es una consecuencia de un conjunto dado de sentencias, es decir, en saber hacer deducciones y pruebas de independencia". ${ }^{6}$

Desde el punto de vista lógico, la expresión 'los que no son lógicos puros' denota la clase complementaria de la denotada por la expresión 'los que son lógicos puros'. Los que no son lógicos puros no deberían interesarse en "la lógica por sí misma", pero esto todavía no permitiría saber en qué pueden interesarse. Muchos de estos no se interesan por la lógica en absoluto: el no ser lógico puro no implica todavía ser lógico (siquiera) "impuro". Supongamos, sin embargo, que los que no son lógicos puros siguen teniendo intereses lógicos. Estos serán los que, según Mosterín, se interesan por las aplicaciones de la lógica, pero aun esto me parece dudoso en todos los casos.

Admitamos que los filósofos sean lógicos "no puros". Puede interesarles la lógica sin que les interesen necesariamente sus aplicaciones a modo de un ejercicio consistente en resolver problemas de acuerdo con reglas. Estas aplica. ciones constituyen una tarea lógica, llevada a cabo por lógicos (que, ipso facto, dejan de ser "puros", pero que siguen siendo lógicos) y por no lógicos, incluyendo quienes aprenden lógica, sea para convertirse oportunamente en lógicos puros o para seguir siendo lógicos "aplicados".

La lógica aplicada es una cosa; la aplicación de la lógica para plantear, aclarar o resolver problemas filosóficos es otra; la reflexión filosófica sobre la lógica - llamada a veces "filosofía de la lógica" y a veces "lógica filosófica", una tercera. A los filósofos - a menos que sean, por su cuenta y riesgo, "lógicos puros" puede no interesarles "la lógica por sí misma", pero puede seguirles interesando la lógica sin que por ello hagan lógica aplicada.

Por lo demás, cabe preguntarse si los lógicos puros, al in.

- Loc. cit. 
teresarse por la teoría lógica y por los fundamentos de la lógica, no es interesan por cuestiones a las que pueden prestar asimismo atención algunos filósofos. No es fácil responder a esta pregunta con toda la claridad apetecible en virtud del incierto y discutido status de la llamada "lógica filosófica" o de la "filosofía de la lógica".

Por una parte, parece que todo problema de filosofía de la lógica pueda tratarse como un problema de lógica (o de metalógica). Al formular, como hace Hilary Putnam, la cuestión de si es o no "indispensable" cuantificar entidades abstractas (números, clases, conjuntos, etc.), ${ }^{7}$ cabe reaccionar diciendo que la pregunta huelga: no hay inconveniente en erigir un sistema lógico donde se cuantifiquen entidades abstractas (por ejemplo, y especialmente, clases) y otro donde no se cuantifiquen dichas entidades (o alguna clase de ellas) y se proceda a cuantificar solamente individuos o "entidades concretas". No se requiere ninguna decisión "extra-lógica" y no se plantea, por tanto, ningún problema de filosofía de la lógica.

Por otra parte, se puede afirmar que dicha pregunta no es ociosa desde el punto de vista filosófico, ya que una respuesta a la misma va ligada a la adopción de una posición sobre el sentido que tiene 'hay...' o '... existe" ${ }^{8}$ para las llamadas "entidades abstractas" y, por si fuera poco, está condicionada por la posibilidad de aplicar o no un sistema lógico, o matemático, a determinadas ciencias. Aun si se mantiene que el sentido de 'hay ...' o de '... existe' no es el mismo para entidades abstractas que para entidades concretas - por ejemplo, que para las primeras toda cláusula existencial es una convención-, se adopta una posición "extra-lógica", a saber, una que conlleva cuestiones relativas no solamente a estructuras lógicas, sino también a sístemas se-

7 Hilary Putnam, Philosophy of Logic (New York-Evanston-San FranciscoLondon, 1971), especialmente pp. 57 y ss.

8 Para simplificar prescindo de que 'hay...' puede interpretarse de modo distinto que '... existe'. 
mánticos, condiciones epistemológicas, supuestos ontológicos, etc. Se alegará que desde el punto de vista lógico no es necesario responder a ninguna de estas preguntas ni adoptar ninguna de las aludidas posiciones, ya que los sistemas lógicos son puros juegos formales -o en vista de que, como escribió Wittgenstein, "la lógica debe hacerse cargo de sí misma". ${ }^{9}$ Pero la idea de los sistemas lógicos como puros juegos formales no es una idea lógica ni, estrictamente hablando, metalógica: es una idea sobre la lógica y puede ser considerada como una parte de una posible filosofía de la lógica.

Entre la filosofía de la lógica y la aplicación de la lógica para el planteamiento, aclaración y posible solución de problemas filosóficos, hay una zona intermedia que, como todas las de esta especie, pertenece a todos y a nadie, y no se sabe bien si es filosofía de la lógica, lógica filosófica o, como es lo más probable, un conjunto de técnicas analíticas que afectan a muy variados problemas, algunos de los cuales son calificados de "lógicos" en el sentido de que son planteados por nociones que se emplean, o presuponen, en la lógica, otros son semánticos, otros lingüísticos, ${ }^{10}$ otros epistemológicos, otros ontológicos, etc. Considérese la lista dada por Strawson: ${ }^{11}$ forma general proposicional, referencia $y$ predicación, funciones veritativas y condicionales, significado y uso, significación y necesidad, verdad, categorias. Se puede aumentar esta lista o acortarla (algunos, por ejemplo, dirán que 'categoría' no es un término "estrcitamente lógico" a menos de usarse como término técnico dentro de una construcción lógico-matemática que incluya las nociones "subordina-

9 "Die Logik muse für sich selber sorgen" (Notebooks 1914-1916, 22.8.14).

10 Con los términos 'semántico' y 'lingüístico' ocurre algo parecido a lo que sucede con 'lógico'; cabe hablar de semántica filosófica y de filosofía lingüistica (o tal vez lingüística filosófica) sin que se sepa a ciencia cierta de qué se habla, porque en cada caso se trata de zonas intermedias, cambiantes e "interdisciplinarias". Pero el que no se puede circunscribir siempre exactamente un "sujeto" no quiere decir que no lo hay; puede querer decir que hay "demasiado de él".

${ }_{11}$ P. F. Strawson, ed., Philosophical Logic (Oxford, 1967), "Introducción", pp. 3-14. 
das" de clase y conjunto), o modificarla parcialmente, pero en la gran mayoría de casos las nociones de referencia están en el mismo centro del análisis filosófico a la véz que son nociones de interés lógico $\longrightarrow$ que son las nociones de interés lógico que al mismo tiempo son más susceptibles de tratamiento filosófico. Como en esta "zona intermedia" pueden descubrirse algunos de los problemas básicos de lo que tradicionalmente se llamó "filosofía teórica", es razonable concluir que hay una relación estrecha entre ciertos aspectos de la filosofía y ciertos aspectos de la lógica, de modo que en. tonces la lógica no es ni propedéutica ni parte o rama de la filosofía: es una especie de locus donde pueden hallarse pro. blemas filosóficamente básicos. Esto sugiere la especial importancia que tiene "la" lógica para la filosofía, y el que se pueda hacer filosofía con poca, o ninguna, biología molecular o con poca, o ninguna, historia de la literatura, pero que no se pueda hacerla sin alguna lógica. Ello vale especialmente para los modos analíticos de hacer filosofía, o cuando menos para su parte metodológica. No es menester que "la" lógica sea el fundamento de lo filosofía, o de la metodología, analítica, primero porque no se sabe bien cómo ésta podría "fundarse" en la lógica, y segundo porque lo que se llama "la lógica" no es "la lógica pura", sino simplemente un conjunto de nociones y operaciones con las que nos familiarizan los "lógicos puros" y que, como filósofos, podemos tomarnos la libertad de mirar "impuramente", for: mulándonos cuestiones tan poco estrictamente lógicas como "¿Qué clase de referencia tienen, si alguna tienen, los términos generales?” o “¿Qué clase de predicado es 'es verdadero'?"

De este modo, la filosofía, tal como aquí la entiendo, mantiene variadas relaciones con lo que seguiré llamando "lógica". Las relaciones serán más o menos estrechas según se concluya que es menester "regimentar" más o menos el lenguaje filosófico. Una regimentación máxima llevaría a identificar filosofía con lógica - lo que equivaldría, a la 
postre, a despachar la filosofía-. Una regimentación nula sería filosóficamente perniciosa. Una regimentación parcial para efectos determinados parece más a propósito. Tal regimentación parcial puede entenderse de varias maneras. Una, propuesta por Quine, equivale a una regimentación parcial máxima de acuerdo con la regla de que "en general, puede considerarse que los sistemas canónicos de notación lógica no son notaciones completas para discurrir sobre temas especiales, sino notaciones parciales para discurrir sobre todos los temas": ${ }^{12}$ no más, pero tampoco no menos, "lógica" de lo que sea necesario. Otra, propuesta por autores especialmente interesados en el análisis del lenguaje corriente, equivale a una regimentación parcial mínima; a veces parece desaparecer por completo sepultada bajo el alud de la "lógica informal", pero aún ésta sólo puede ser entendida en relación con sistemas de regimentación y codificación uno de los cuales es la lógica formal; ésta hace con ciertas constantes como ' $y$ ', 'o' o 'no' algo que el lenguaje corriente no tiene por qué hacer, y la lógica hace con las mismas constantes algo que no tiene por qué hacer el lenguaje corriente.

La "lógica por encima de todo" no lleva a la filosofía a ninguna parte, salvo a la propia lógica, y cuando todavía queda algo de filosofía en esta lógica lleva a la idea de que "lógica" y "pensamiento racional" son lo mismo -idea que es la única proposición formulable en esta "filosofía como lógica"-. La "impotencia de la lógica" no lleva a ninguna parte ni a la filosofía ni a la lógica; paradójicamente, la doctrina, o predicación, de la "impotencia de la lógica" tiene de común con "la lógica por encima de todo" el presuponer el dilema "o lógica o no lógica", amén de la identificación de la lógica con el pensamiento racional, pero en virtud de su adopción de la "no lógica" (que tal vez llame "prelógica"), arrastra consigo hacia la nada —que es don-

12 Willard van Orman Quine, Word and Object (Cambridge-New YorkLondon, 1960), pág. 160. 
de, de todos modos, quiere llegar- a la vez lógica y razón, es decir, toda clase de argumentación, incluyendo la que está mínimamente regimentada. En vista de estas poco apetitosas perspectivas, es más razonable seguir manteniendo en filosofía la lógica, aunque no "por encima de todo", y, además, o concomitantemente, admitir muy variadas formas de argumentación racional, incluyendo las que destacan el hecho de que los argumentos son parte de un proceso de comunicación lingüística." Los argumentos "puramente lógicos" no son, propiamente hablando, argumentos: son partes de algún pro. ceso de inferencia o deducción lógicas. Por eso los argumentos filosóficos, sin dejar de ser lógicos en el sentido de "no pecar contra la lógica" (lo que, a la postre, quiere decir sim. plemente, no ser inconsistentes), no son sólo lógicos; como ha indicado Max Black, no hay muchos casos, de haber alguno, en que la crítica puramente lógica de un argumento filosófico pueda tener gran interés "como crítica de un auténtico argumento filosófico" 14 -lo que equivale a dudar que lo que haya de malo en un argumento semejante sea siempre un sofisma o "falacia formal"."

Las "relaciones especiales" de la filosofía con la lógica han sido más o menos intensas; durante el siglo actual, y especialmente por parte de algunas de las corrientes del lla-

13 Esto puede hacerse de varias maneras: atendiendo a propósitos y modelos de argumentos y a las posibles conexiones entre razonamientos lógicos y la práctica -inclusive la práctica "cotidiana"- de la argumentación, como lo hace S. E. Toulmin en The Uses of Argument (Cambridge, 1958); examinando ciertas peculiaridades de los argumentos corrientemente usados por los filósofos, como las estudia John Passmore en Philosophical Reasoning (New York, 1961); atendiendo máximamente a la comunicación y a las situaciones humanas de la comunicación, como lo ha hecho Chaïm Perelman en varias obras relativas a la "nueva retórica" (Rhétorique et Philosophie [Paris, 1953]; Traité de largumentation, 2 vols. [Paris, 1958]; resumen de sus ideas en el artículo "The New Rhetoric: A Theory of Practical Reasoning", en Great Ideas To-Day (1970); estudiando argumentos legales e inferencias prácticas, al modo de R. M. Hare (por ejemplo, en Practical Inferences [London-New York, 1971]).

14 Max Black, "Logic and Ordinary Language", en Language, Belief, and Metaphysics (Albnary, N. Y., 1970), ed. Howard E. Kiefer y Milton K. Munitz, p. 47.

15 Art. cit., pág. 48. 
mado "giro analítico", han sido particularmente estrechas. Como ocurre a menudo en estos casos, se producen reacciones adversas que llevan a los dos miembros de la relación a sacar a la luz del día hostilidades latentes y a preguntarse si no sería mejor el divorcio, o cuando menos la separación legal. Como, por lo general, se repara primero en las fallas ajenas que en las propias, no pocos filósofos han empezado a preguntarse qué ha "fallado" en la lógica, y si así es qué puede proponerse en su lugar. ${ }^{16}$

Si ha fallado "la lógica", entonces se puede recurrir, han dicho algunos - volviendo del revés las críticas antipsico. logistas de Frege y Husserl, y las críticas antipsicologistas, antihistoricistas y anti-"naturalistas" del último- a la psicología, o a la historia, o a ambas. Ni la psicología ni la historia son explicables mediante la lógica formal. Los lógicos formales estarían de acuerdo con esto, pero no con lo inverso: la lógica formal es explicable mediante la psicología y la historia —o la sociología-. Por 'ser explicable' se entiende las más de las veces 'estar originado en'. Se dice, así, que la lógica en cuanto disciplina formal, o conjunto de disciplinas formales, tiene un origen histórico, y específicamente que el propio concepto de lógica formal y la idea de lógica formal han surgido de la necesidad de dominación y control sobre la realidad. ${ }^{17}$ De este modo, la explicación genética se identifica con la "justificación", y el conjunto se encaja dentro del marco de una llamada "lógica dialéctica". La última, sin embargo, no es, propiamente hablando, una lógica, sino una doctrina, o un principio. Es posible for. malizar los últimos, como ha hecho F. G. Asenjo para la dialéctica hegeliana, donde "no se usa el principio dialéc-

16 En este artículo me refiero exclusivamente a la lógica formal y no a la titulada "lógica informal". Cualesquiera que sean los méritos de la última, no puede sustituir a la lógica formal y, por tanto, no resuelve los problemas que se plantean con respecto a aquella.

17 Véase, por ejemplo, Max Horkheimer y Theodor W. Adorno, Dialektik der Aufklärung (Amsterdam, 1947). 
tico como regla de inferencia, sino como regla de forma. ción". ${ }^{18}$

La falla principal de estas críticas de "las fallas de la lógica" reside en que se pasa del reconocimiento de que la lógica no constituye una serie de principios de conocimiento a la afirmación de que tiene que fundarse en prin. cipios o factores extra-lógicos -no sólo "originarse" en ellos-, con lo cual se niega la neutralidad de la lógica. Pero si la lógica no es neutral, entonces debe constituir una serie de principios de conocimiento, posición incompatible con la precedente. Análogas fallas se revelan en los intentos de mostrar que hay estructuras lógicas "innatas" en un organismo biológico, y específicamente en el sistema neural humano; aunque hubiese tales estructuras, ello no probaría que no son neutrales. La relación isomórfica entre las su. puestas estructuras "innatas" y las estructuras lógicas propias de los sistemas formales no constituye ninguna prueba de que las últimas estén fundadas en las primeras.

Un dictamen hoy bastante extendido, y más aceptable que los reseñados antes, es el de que ha fallado el "racionalismo extremo", el cual se ha apoyado o en un sistema metafísico de supuestas verdades eternas, las cuales en tanto que ver. dades de razón son verdades últimamente lógicas, o bien en "la lógica", que se supone expresa formalmente el antedicho sistema de verdades eternas. Ortega y Gasset puso de ma. nifiesto, hace ya tiempo, que "el pensamiento" se ha ocul. tado durante siglos en "la lógica", la cual "suplanta la infinita morfología del pensamiento por una sola de sus formas: el pensamiento lógico", ${ }^{18}$ siendo "incalculable el poder de

18 F. G. Asenjo, "Lógica dialéctica", Teorema, 1 (1971), 7-13 (publicado originariamente en inglés, en Logique et Analyse, 32 [1965]. Véase también la nota aclaratoria de Asenjo en Teorema, 6 (1972), 193-94. Asimismo, Michael Kosok, "The Formalization of Hegel's Dialectical Logic", en Hegel: A Collection of Critical Essays, ed. Alasdair McIntyre (Garden City, N. Y., 1972), pp. 237-87; originariamente publicado en International Philosophical Quarterly, 6 (1966).

18 José Ortega y Gasset, “Apuntes sobre el pensamiento: su teurgia y su demiurgia", Logos, 1 (1941), recogido en Obras completas, V. 527. Ortega 
ocultación que durante dos milenios ha ejercido este imperativo casi religioso de 'logicidad' ". ${ }^{20}$ Con esto no se trata de rebajar la importancia del "pensamiento lógico" (el propio Ortega apuntó que la posibilidad de no considerar la lógica como patrón absoluto de verdad ha corrido parejas con el aumento de refinamiento de la lógica y de sus técnicas) ${ }^{21}$ ni tampoco como una manifestación de relativismo e historicismo.

Podrían incorporarse fácilmente estas ideas a las expresadas por varios autores que, como S. Toulmin, han manifestado que "en la ciencia, no menos que en la filosofía, la preocupación exclusiva por la sistematicidad lógica ha ido en detrimento tanto de la comprensión histórica como de la crítica racional". ${ }^{22}$ Según Toulmin, todas las teorías filosóficas que se propusieron desde muy pronto para resolver los desacuerdos entre los hombres a base de un "tribunal imparcial de la razón" llevaron a algún "sistema simple y permanente de ideas y creencias", cuyo modelo básico estuvo constituido por la lógica y la geometría: "De este modo, la 'objetividad', en el sentido de imparcialidad, se equiparó a la 'objetividad' de las verdades intemporales; los méritos racionales de una posición intelectual se identificaron con su coherencia lógica, y el patrón que el filósofo descubrió para medir la racionalidad humana resultó ser su habilidad para reconocer, sin más argumentos, la validez de los axiomas, implicaciones formales y necesidades lógicas de que dependían las propuestas del sistema autoritario" ${ }^{23}$ (o sistema que ejerce la función de autoridad suprema y "decisiva"), sin importar para el caso que no siempre fuese obligatoria la equiparación de la racionalidad cọn la logicalidad

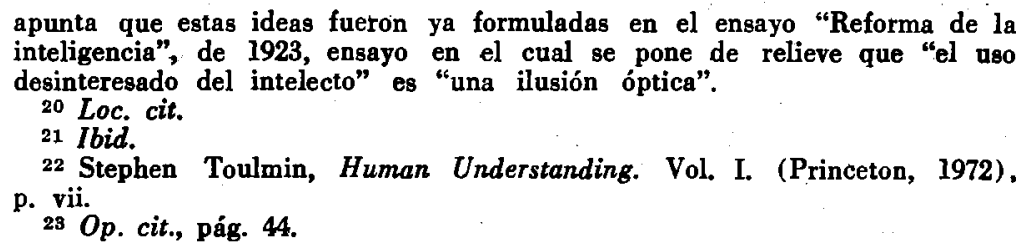


(o logicidad), como lo muestran numerosos ejemplos históricos tan numerosos que, en puridad, uno empieza a pensar si las expresiones 'durante milenios' y 'desde muy pronto' no serán más bien retóricas.

Ninguna de las opiniones últimamente citadas lleva a concluir que la lógica no es neutral; por el contrario, en varios casos se insiste en que tiene que ser neutral justa y precisamente porque las consideraciones lógicas no permiten ni derivar de ellas teorías ni decidir entre dos o más teorías; en este sentido, "la lógica" es "impotente", y lo es asimismo "la razón" a veces identificada con aquélla.

Pero aunque la lógica - como sistema de relaciones formales- no es lo que se llama "la razón", y ninguna de ellas constituye "el Tribunal" ante el cual hay que justificar todos los asertos básicos, y aunque las razones que se dan para adoptar una teoría, e inclusive para adoptar un determinado lenguaje formal con el fin de expresarla, sean extralógicas, la lógica es un ingrediente que figura, en mayor o menor proporción, en los asertos, y especialmente en los sistemas de asertos, o teorías, que se formulen. Si hemos de creer lo que Platón nos cuenta, Sócrates se dirigía a veces a . sus interlocutores preguntándoles si preferían un argumento o un relato. En general, los interlocutores preferían el relato (lo que daba a Platón la oportunidad de abstenerse de producir un argumento). Aquí no ofrezco ni un argumento ni un relato, sino una serie de comparaciones e imágenes.

$\mathrm{Ni}$ "la lógica" ni "la razón" constituyen "el Tribunal", pero no porque los tribunales hayan dejado de existir, sino más bien porque se han multiplicado, e independientemente de que nos gusten o no, hay que convenir en que es mejor tener varios que uno solo, y mejor aun tener varios sin que ninguno de ellos reclame ser el supremo, porque aunque se suponga que un tribunal supremo decide sólo en algunos casos, resulta que son los más graves. Cierto que la pluralidad de tribunales puede prestarse a confusiones, porque entonces habrá una pluralidad de juicios y de sentencias, y ello 
sin que haya necesariamente una correspondiente pluralidad de disputas, esto es, sin que cada disputa, o tipo de disputa, tenga su correspondiente tribunal. Disputas las hay de muchas clases, y algunas son vistas y juzgadas por el tribunal que se estime en cada caso más apropiado, pero hay otras que son vistas y juzgadas por varios tribunales, que pronuncian distintas sentencias, y podemos preguntarnos entonces si hay que atenerse sólo a una de éstas o si es posible tener en cuenta más de una.

Creo que es lo último, y que no por ello debe concluirse que habrá siempre incompatibilidad entre los distintos jui. cios. La compatibilidad no se debe, por otro lado, a que subyaciendo las disputas haya "la Disputa" o subyaciendo los juicios haya "el Juicio". Lo que hay entre disputas, tribunales, juicios, sentencias, etc., son relaciones más o menos flexibles - relaciones de vecindad, y alguna que otra vez de familia-. Esto no exige que todos los jueces y litigantes hablen el mismo lenguaje, pero requiere que hablen lenguajes que puedan traducirse, aunque sea parcialmente, y en los cuales puedan entenderse y comunicarse los interesados, aunque sea también sólo en parte, y hasta con ayuda de algunos gestos, más o menos felizmente interpretables. Si a la trama de estos "lenguajes" se la califica de "Razón" (con mayúscula), no se habrá ganado todavía gran cosa, y hasta se puede perder bastante, porque se le sobrecarga de trabajo y puede terminar exhausta. Llamémosla, de momento, "razón" (con minúscula). Este artificio tipográfico no permite saber en qué consiste, pero sugiere que hay ahí algo que refleja lo que se suponía había en aquella "Razón" - la aspiración a la objetividad y a la universalidad-, aunque ya bastante deshinchado y con un valor más bien pragmático. La razón en minúscula no es una razón ya constituida, morando eternamente en un universo trascendente e independiente de los problemas reales y de las condiciones reales de operación, con sus intereses, tensiones y conflictos; es una razón que se va constituyendo. 
El principal mérito de la "trama" llamada (para simplificar) "razón", reside en su valor de cambio, que se modifica, pero no al punto de resultar inusable. Como sucede con las divisas, la razón por sí misma no parece valer gran cosa, pero sirve para mucho, ya que asegura cierta "objetividad" y permanencia en los intercambios. La razón no es un signo completamente arbitrario en un sentido a como no es completamente arbitraria una divisa, ni siquiera cuando sufre serias presiones inflacionarias, o deflacionarias. La razón no es, en suma, ninguna "realidad", sino un conjunto de funciones. En este sentido puede decirse que se desarrolla en el curso de la historia. ${ }^{24}$ Lo que en el curso de la historia hacen los hombres va constituyendo la razón, y a la vez ésta permite contrastar, comparar o discutir lo que se hace, o piensa.

Como uno de los ingredientes con que está fabricada la divisa aludida es la coherencia formal - la cual no garantiza la verdad de ninguna proposición, pero sirve de control para evitar falsificaciones demasiado gruesas en el manejo de proposiciones-, puede decirse que "la lógica" es un componente de "la razón". Desde este punto de vista, la lógica es tanto más necesaria cuanto más se requiere una regimentación del lenguaje. Por eso la lógica da la impresión de que, a diferencia de otros lenguajes, "puede hacerse cargo de sí misma". Sin embargo, esta impresión es sólo una de las caras que ofrece la relación entre la lógica -mejor dicho, la actividad lógica - y las demás actividades.

$\mathrm{Si}$ se entiende la lógica como "lógica matemática" en el sentido de una disciplina que en vez de limitarse a la formación y manipulación de sistemas simbólicos y juegos formales, construye - para ser justos: construye también-

24 ¿Cómo? No creo que sea acumulativamente ni tampoco anárquicamente. Es posible que sea en parte dialécticamente, pero sin seguir un esquema dialéctico rígido, cuya aceptación equivale a la de una Razón trascendente; 'dialéctico' quiere decir aquí 'conflictivo'. Lo más probable es que la razón se desarrolle "pragmáticamente", con periodos más o menos estables y crisis revolucionarias, que, por lo demás, pueden ser de muy larga duración. 
"modelos" para la investigación empírica y, en último término, para todas las investigaciones y actividades humanas, ${ }^{25}$ tendremos en ella algo más que un instrumento que puede ser aplicado, más o menos mecánica y dócilmente, al plan. teamiento, aclaración y solución de cuestiones de toda clase, tanto en la ciencia como en la práctica humana. De un modo similar a lo que ocurre con la matemática en las ciencias, la lógica puede conducir a formular, reformular, y hasta descubrir, problemas. Al efecto sienta criterios de adecuación muy radicales: no sólo contribuye a las pruebas, sino también a probar lo que ha de valer como prueba. Puede decirse que la lógica transforma los problemas y con ello ayuda a "la razón" a constituirse.

Al mismo tiempo, en el curso de la citada actividad, la lógica se transforma a sí misma, cosa que no sería posible si los problemas "extralógicos" no influyeran a su vez sobre la formación de modelos lógicos. No sólo las cuestiones filosóficas no son ajenas a la lógica, sino que la lógica no es tampoco ajena a dichas cuestiones. Quienes se manifiestan invariablemente en favor de un tratamiento lógico de problemas filosóficos, lo entienden de un modo unilateral: como si la lógica determinara y controlara tales problemas. Si se quiere seguir considerando la lógica como una herramienta, habrá que concluir, pues, que ésta se modifica de acuerdo con el trabajo que se hace con ella.

25 Debo a mi colega, el profesor George Weaver, el haber llamado mi atención sobre el poder "normalizador" de la lógica matemática en toda clase de investigaciones, incluyendo las filosóficas. 
'Logic' is taken here exclusively as 'formal logic', not as 'informal logic'. It is asserted that although logic is neutral, in the sense at least of being topic-free, it can be used in the formulation and treatment of philosophical problems. Furthermore, some philosophical problems may be raised in logic.

This does not mean that logic is a part of, or a propedeutic to, philosophy. There are between logic and philosophy "special relations". These have been particularly intense in some philosophical schools, but questions have been raised as of recent about whether patterning of philosophical thought on logical structures and/or concepts has not gone too far. It has been pointed out, moreover, that if logic has "failed" (in philosophy), reason has failed as well.

The author does not think that either logic or reason has failed in philosophy. What may have failed is the idea that logic is to be equated to Reason, and the latter is to be compared to a kind of "Supreme Court" which has the power to give a final decision to all controversies. There is no reason why logic should be equated to Reason, and there is no reason why Reason should be capitalized. Rather than Reason there are "reason", multitude of "courts" to which discussants appeal and which are not necessarily unrelated one to the other, but are not subordinated to one "Grand Court of Reason".

Formal logic is one of the ingredients in these various "courts". Although it is not the court member that ultimately "decides", it is the one which tends to "unify" - the degree of unification depending about the subject matter discussed. In this sense logic is a kind of "normalizer" - a point that is often made when the normalizing power of formal logic is emphasized.

To be sure, there is no such thing as "the logic", nor is any formal logic immune to changes. This, however, rather than discourage the "special relations" between philosophy and logic, should tend to intensify them. 